\title{
Nordiques
}

39 | 2020

Varia

\section{Les perceptions journalistiques du « modèle suédois » en France (1990-2020)}

\section{Christophe Premat}

\section{(2) OpenEdition}

\section{Journals}

Édition électronique

URL : http://journals.openedition.org/nordiques/609

DOI : $10.4000 /$ nordiques.609

ISSN : 2777-8479

Éditeur :

Association Norden, Bibliothèque de Caen la mer

Référence électronique

Christophe Premat, «Les perceptions journalistiques du « modèle suédois » en France (1990-2020) », Nordiques [En ligne], 39 | 2020, mis en ligne le 01 novembre 2020, consulté le 13 mars 2021. URL: http://journals.openedition.org/nordiques/609; DOI : https://doi.org/10.4000/nordiques.609

Ce document a été généré automatiquement le 13 mars 2021.

Nordiques 


\title{
Les perceptions journalistiques $\mathrm{du}$ « modèle suédois » en France (1990-2020)
}

\author{
Christophe Premat
}

\section{Introduction}

1 Il n'est pas de projet de grande réforme sociale et économique à l'heure actuelle en France sans que l'exemple de la Suède soit cité. Dans les années 1980, le modèle suédois figurait pourtant très peu dans le répertoire politique français ${ }^{1}$. Si à la fin des années 1980 , on s'intéresse aux relations sociales en Suède, la crise économique du début des années 1990 de ce pays s'est accompagnée par un changement profond de paradigme. Ainsi, la Suède devient petit à petit une référence pour promouvoir une efficacité des politiques publiques et des réformes du marché du travail. Lors de son discours à Göteborg le 17 novembre 2017, le président Macron déclarait : « Le modèle suédois est connu et est une source d'inspiration pour la France. Vous avez réussi à respecter cet engagement fort envers l'équité et l'ambition sociale. Et ce n'est pas tout: vous avez également réussi à faire baisser votre taux de chômage, vous avez également réussi à créer plus d'emplois; c'est exactement la même chose que nous souhaitons réaliser $»^{2}$. En s'adressant aux autorités suédoises, Macron célébrait les bons résultats économiques de la Suède qui permettaient de soutenir les dépenses sociales. En d'autres termes, le modèle suédois est devenu une boussole dans l'agenda politique français avec cette idée qu'une bonne compétitivité fût nécessaire pour financer une protection sociale adaptée.

2 La réforme des retraites portée par le gouvernement français à la fin de l'année 2019 a prouvé que la multiplicité des références au modèle suédois pouvait être problématique selon les politiques publiques envisagées ${ }^{3}$. Dans le foisonnement récent de ces références, nous aimerions montrer comment la transformation radicale de la Suède au début des années 1990 est en fait mobilisée dans le discours journalistique français pour 
justifier un nouvel élan. Emile Chabal proposait d'analyser les évolutions politiques et sociales de la France d'après-guerre à partir de trois récits matriciels, un récit néorépublicain centré sur la nécessité de préserver la laïcité, un récit postcolonial traitant de l'intégration et de la mémoire des différents groupes sociaux vivant en France et un récit libéral habité par le souci de retrouver un dynamisme du marché et une décentralisation efficace ${ }^{4}$. Le modèle suédois pourrait être un détour pour critiquer l'inefficacité du modèle politique et social français jugé trop loin des réalités économiques de la mondialisation ${ }^{5}$. Yohann Aucante a pour sa part montré comment la version classique du modèle suédois avait innervé les essais politiques en France depuis les années $1960^{6}$; or, au moment de la crise du modèle social-démocrate suédois au début des années 1990, on remarque que la référence à cette expression dans la presse française devient de plus en plus ambivalente et polysémique ${ }^{7}$. Auparavant, dans les années 1960 et 1970, de nombreux essayistes et journalistes français avaient publié des ouvrages vantant un modèle social-démocrate égalitaire doté d'un système de protection sociale efficace avec des syndicats puissants ${ }^{8}$. La presse française commençait alors à se faire timidement l'écho de ce modèle auprès du grand public ${ }^{9}$.

C'est en fait dans les années 1990 que ces références à ce modèle commencent à se multiplier au moment où le pays traverse une crise juste avant son entrée dans l'Union européenne. Nous aimerions considérer dans la présente étude la mobilisation de ces références journalistiques qui diffusent plus largement l'expression " modèle suédois » dans l'opinion publique. Les articles des quotidiens nationaux, régionaux et des magazines hebdomadaires français commentent l'actualité politique et notamment les discours présidentiels. Ils ajoutent également des interprétations que ce soit par le biais d'éditoriaux ou d'articles de fond. Autrement dit, il convient d'analyser de manière systématique les invocations de ce "modèle suédois » qui en font un " maître mot »10 du discours journalistique et qui est identifié à une "Middle way " ${ }^{11}$. De cette manière, grâce au moteur de recherche journalistique Nexis Uni, il est possible d'identifier les sources des articles faisant référence aux expressions consacrées "modèle suédois ", « modèle scandinave » et « modèle nordique ». Les journalistes ont en effet participé à la valorisation de ce modèle dans l'opinion publique française. En l'occurrence, ils jouent un rôle fondamental dans la promotion de ce que l'on appelle les marques nation, soit nation branding en anglais ${ }^{12}$.

\section{Recherches antérieures et méthodologie retenue}

L'invocation du modèle suédois fait certainement partie des mythologies au sens développé par Roland Barthes, à savoir de mécanismes sémiologiques permettant d'activer une forme de tautologie. «On pourrait dire que la langue propose au mythe un sens ajouré. Le mythe peut facilement s'insinuer, se gonfler en lui : c'est un vol par colonisation $»^{13}$. La signification est comme parasitée, la langue ne pouvant pas empêcher ce processus d'interprétation. Dans cette perspective, l'analyse mythologique permet de se concentrer sur la circulation d'une formule toute faite dans le monde politique et journalistique. Pour sa part, Cornelius Castoriadis avait défini la notion d'institution imaginaire de la société fondée sur l'élaboration de significations imaginaires sociales donnant un sens aux actions des individus ${ }^{14}$. De facto, la signification dépasse la fonctionnalité linguistique pour intégrer un ensemble de représentations sociales qui sont plus ou moins consciemment activées par les 
individus ${ }^{15}$. Dans cette optique croisant les approches de Barthes et de Castoriadis, la notion de modèle suédois se traduit par la mobilisation de représentations sociales diverses, d'affects (proximité ou rejet) et d'intention (inspiration ou au contraire exclusion). Ainsi, le modèle suédois est toujours pris dans des énoncés non neutres activant une forme d'assertion. Le modèle est une image qui s'impose, un élément indiscutable qui a existé, qui perdure et permet d'accentuer un contraste. Il semble que, dans les invocations journalistiques du modèle suédois, se glissent presque systématiquement des métaphores et des descriptions de type culturel marquant une tendance à l'essentialisation. Le modèle peut s'imiter ou se copier, il peut à la limite inspirer, mais il ne laisse jamais indifférent.

5 Gilles Vergnon a montré justement que l'intérêt pour la Suède s'était limité à quelques passeurs jusqu'à la Seconde Guerre mondiale. Ce n'est qu'à partir du milieu des années 1930 qu'une série de publications traite de la Suède, esquissant une voie possible au moment du choc entre des systèmes autoritaires à vocation totalitaire ${ }^{16}$. Si on regarde de plus près la situation avec notamment des sources suédoises, on se rend compte que le modèle suédois est promu à partir de l'installation des gouvernements sociauxdémocrates. De l'aveu même des opposants à Per-Albin Hansson, les sociauxdémocrates suédois avaient récupéré une formule-clé (folkhem) élaborée par des penseurs conservateurs tels que Rudolf Kjellén ${ }^{17}$. A fortiori, cette formule a permis d'installer la conception d'un État-providence assurant un confort individuel minimal à tous les individus.

6 Dans les travaux sur les transferts culturels inspirés par Michel Espagne, Martin Kylhammar s'est intéressé aux récits de voyage et aux transferts culturels qui ont permis de colporter des visions et des modèles ${ }^{18}$. Serge de Chessin est l'un des premiers à s'émerveiller des ressources de la société suédoise tentant de garantir le bien-être pour tous ${ }^{19}$. Avec l'arrivée des nazis au pouvoir, Serge de Chessin est celui qui effectue dans son ouvrage Les clefs de la Suède une description de l'émergence de la société social-démocrate avec le folkhem ${ }^{20}$. Cette présentation n'est pas partagée par Christian de Caters qui au contraire insiste davantage sur l'exotisme d'une société qui ne peut prétendre à être un modèle ${ }^{21}$. Par la suite, Émile Schreiber et Paul Planus appuient à la fin des années 1930 l'entreprise de Chessin pour remettre à l'honneur cette idée de modèle ${ }^{22}$. Enfin, Martin Kylhammar a montré que le curseur s'est déplacé sur un portrait de l'homme du nord ${ }^{23}$. Selon Martin Kylhammar, depuis les années 1980, l'évocation de la Suède semble prise dans une dialectique entre modèle et contremodèle. Il suggère également le fait que le modèle suédois soit de plus en plus intégré à une perception plus globale du modèle nordique en France, ce que nous souhaitons tester de manière empirique à partir de l'apparition des termes "modèle suédois ", "modèle nordique" et "modèle scandinave" dans les quotidiens nationaux et régionaux français. L'un des premiers analystes à avoir écrit un ouvrage intitulé $L e$ modèle suédois était Jean Parent qui, au début des années 1970, présentait clairement les caractéristiques de la social-démocratie incarnée alors par la vague de compromis sociaux avec les syndicats et un parti dominant l'échiquier politique ${ }^{24}$. En réalité, les années 1960 et 1970 ont vu la multiplication des essais portant sur la Scandinavie et en particulier la Suède qui ont contribué à la cristallisation du mythe suédois ${ }^{25}$.

7 Dans les années 1980, les analyses des politiques suédoises demeurent rares à l'exception de certains travaux sur la social-démocratie et l'État providence; on remarque également plusieurs publications scientifiques évoquant un des aspects 
spécifiques du modèle suédois ${ }^{26}$. Puis, les années 1990 voient l'éclosion de plusieurs livres sur ce modèle avec la parution de l'ouvrage de Jacques Arnault ${ }^{27}$. Il faut dire que la crise que traverse la société suédoise à cette époque ne permet plus d'activer une référence globalement positive au modèle suédois. Depuis les années 1990, les références au modèle suédois dans les travaux des chercheurs francophones sont liées à l'évolution des systèmes de protection sociale suite à la crise de l'État-providence ${ }^{28}$. L'ouvrage de Magnus Falkehed en 2003 vient relancer l'intérêt des chercheurs pour les réformes économiques entreprises en Suède depuis l'entrée de cette dernière dans l'Union européenne avec cette fois une présentation plus équilibrée des avantages et des inconvénients ${ }^{29}$.

Récemment, l'ouvrage d'Alain Lefebvre venait discuter la manière dont le Président Macron s'était emparé de ce modèle pour y réaliser des réformes libérales ${ }^{30}$. Cela étant, très peu d'études ont porté exclusivement sur l'invocation de ce modèle dans les journaux français. Les références et les discussions du modèle suédois sont omniprésentes, mais au-delà des études culturelles et de la science politique, très peu de recherches empiriques ont porté sur le discours relayé par les quotidiens. Il est évident que dans ces quotidiens, les prises de parole sont diverses, puisqu'il peut y avoir des éditoriaux, des articles et des dossiers de fond, des débats, des rapports et commentaires et des interventions plus politiques. Cette recherche s'intéresse davantage à la mobilisation du modèle suédois qui peut être convoqué d'une manière globale comme décrivant un éthos fondamental ou au contraire comme "référentiel » des politiques publiques ${ }^{31}$.

9 La méthodologie retenue consiste à repérer de manière quantitative les fréquences des mentions du "modèle suédois» dans les quotidiens et magazines français pour la période 1990-2020 avec une analyse de certains articles décrivant de manière plus précise ce modèle. En mettant en perspective les mentions de «modèle suédois " par rapport à celles de «modèle nordique » et de "modèle scandinave », il est possible de repérer la manière dont le discours journalistique français mobilise et met en perspective ce modèle. Cette méthode a des limites certaines puisqu'il faudrait pouvoir faire appel à des études qualitatives pour étudier avec précision les références journalistiques au modèle suédois. L'avantage de la lexicométrie est qu'elle nous donne une vue d'ensemble sur les titres d'articles incluant cette référence de manière positive ou négative. L'approche lexicométrique est moins centrée sur les locuteurs, elle s'intéresse davantage au contenu des messages diffusés pour pouvoir étudier les échos, les reprises et les associations lexicales ${ }^{32}$. De surcroît, elle suppose une compréhension du discours comme fragment textuel non borné et utilisé dans des contextes différents.

\section{Les références journalistiques à l'expression « modèle suédois »(1990-2000)}

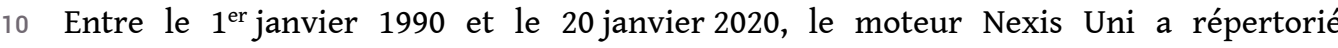
1568 articles en langue française utilisant dans le titre et le contenu l'expression de "modèle suédois ». Ce sont surtout des quotidiens qui tendent à utiliser l'expression dans les articles ou les titres d'articles ( 755 cas) puis viennent les magazines avec 131 occurrences. Le tableau 1 permet de repérer les quotidiens français ayant le plus fréquemment recours à ce syntagme. 
Tableau 1 : repérage de l'expression « modèle suédois » dans les quotidiens français

\begin{tabular}{|l|l|}
\hline Nom du quotidien & $\begin{array}{l}\text { Nombre d'articles faisant référence à l'expression «modèle } \\
\text { suédois » }\end{array}$ \\
\hline Le Monde & 258 \\
\hline Les Échos & 130 \\
\hline Le Figaro & 125 \\
\hline Agence France Presse-Français & 119 \\
\hline Le Figaro Économie & 62 \\
\hline Le Temps & 60 \\
\hline $\begin{array}{l}\text { Sud Ouest et Sud Ouest } \\
\text { Dimanche }\end{array}$ & 50 \\
\hline La Tribune & 43 \\
\hline
\end{tabular}

Source : recherche réalisée sur Nexis Uni (janvier 2020)

11 Parmi les autres quotidiens qui utilisent l'expression, nous trouvons L'Humanité (43 occurrences), Le Point (30 occurrences), des quotidiens régionaux (Ouest-France, 37 mentions, Le Télégramme, 29 occurrences, La Nouvelle République du Centre Ouest, 24 occurrences) et certains quotidiens francophones comme La Presse Canadienne (16 références). Autre indication intéressante relevée dans les métadonnées de Nexis Uni, les quatre personnalités politiques apparaissant avec la référence au modèle suédois dans ces articles sont Emmanuel Macron (83 mentions), François Hollande (46 références), Stefan Löfven (41 occurrences) et Ségolène Royal (37 mentions). Avec l'élection de 2014 et l'arrivée de Stefan Löfven, la notion de "modèle suédois » a été convoquée à de nombreuses reprises pour savoir notamment si ce modèle demeurait toujours social-démocrate. Le Monde se concentre surtout sur l'aspect fonctionnel du modèle tandis que Les Échos s'intéressent à sa viabilité économique.

12 Au début des années 1990, la presse française se concentre sur l'alternance politique avec la fin de la longue hégémonie du parti social-démocrate suédois. Un article du Monde du 17 février 1990 est intitulé «Le modèle suédois malmené " $33^{33}$ tandis qu'un autre en 1991 affiche "La fin du rêve suédois " ${ }^{34}$. L'auteur est un journaliste de tendance centriste qui multiplie ses interventions dans Les Échos et fustige le modèle économique français. Selon lui, le modèle suédois social-démocrate est en train de laisser la place à des privatisations ${ }^{35}$. Françoise Nieto est également une journaliste du Monde qui s'intéresse à la transformation du paysage politique suédois. Dans un court article du 10 mars 1992, elle n'hésite pas à relater le décès de «l'un des pères du modèle social-démocrate", l'ancien ministre Gunnar Sträng qui avait été nommé au gouvernement de Per-Albin Hansson en 1945 et avait servi les différents gouvernements de Tage Erlander ${ }^{36}$. Toute la période 1990-1995 est marquée par des articles décrivant la mutation de la société suédoise avant son entrée dans l'Union européenne à l'instar d'une tribune du journaliste suédois Rolf Gustavsson traduite 
dans Le Monde et évoquant «le chauvinisme du bien-être " ${ }^{37}$. Ce constat est en phase avec les publications françaises sur la Suède à l'instar de l'ouvrage collectif $L a$ fin $d u$ modèle suédois dirigé par Jean-Pierre Durand en 1994 et qui se demandait dans quelle direction le modèle suédois allait évoluer ${ }^{38}$. Dans la presse française des années 1990, le «modèle suédois » est moins évoqué de manière globale comme un modèle sociétal, il est plutôt associé à des politiques précises, par exemple l'alliance stratégique entre Renault et Volvo ${ }^{39}$.

13 Dans les années 1995 et 1996, nous trouvons des articles portant sur l'entrée dans l'Union européenne et la réduction des déficits publics. La notion de modèle est évoquée de manière négative pour insister sur la fin du modèle social-démocrate à l'instar d'un article des Échos du 27 mars 1995 intitulé «La Suède en quête d'une crédibilité européenne - La Suède est condamnée à reconstruire son modèle $»^{40}$. Un éditorial à la Une du Monde du 7 avril 1995 vient sonner le glas du modèle suédois en évoquant la collaboration entre sociaux-démocrates et centristes ${ }^{41}$. Ce quotidien consacre un long dossier sur la Suède et les pays d'Europe du Nord, un article d'Alain Debove s'intitule «La social-démocratie a retrouvé le pouvoir en Europe du Nord» et constate la ressemblance des contextes politiques des pays scandinaves et nordiques connaissant une social-démocratie plus pragmatique ${ }^{42}$. Signe des difficultés passagères liées aux réformes économiques, un article des Échos du 9 novembre 1995 est intitulé «Protection sociale, le 'modèle suédois' à l'épreuve »" ${ }^{43}$. En réalité, la presse française a relayé par la suite en 1996 le souhait du nouveau Premier ministre suédois de l'époque, Göran Persson, de créer un «nouveau modèle suédois $»^{44}$. Cette expression se retrouve dans des titres ou des articles pour évoquer la Suède à l'instar d'un article sur l'égalité des sexes du Point évoquant ce nouveau modèle ${ }^{45}$. L'année 1997 montre l'ambivalence des références au modèle suédois avec tantôt l'évocation du modèle social-démocrate historique et tantôt la nouvelle gestion pragmatique des sociauxdémocrates donnant plus d'importance au marché. Un article du Monde du 19 mars 1997 constate que le modèle suédois a changé, mais qu'il existe toujours ${ }^{46}$. On insiste dans la presse française à la fin des années 1990 sur la mutation du modèle social-démocrate suédois passé vers une décentralisation du marché du travail et des conventions collectives locales. Certains quotidiens sonnent le glas de la socialdémocratie suédoise en évoquant certains scandales comme celui de la stérilisation forcée de certaines populations entre 1932 et $1970^{47}$. L'image de la Suède dans les journaux français connus demeure ambivalente avec des évocations positives et négatives, même si le souhait de Göran Persson est repris pour montrer la possibilité d'avoir une économie plus dynamique et des finances publiques vertueuses.

\section{La découverte du « nouveau modèle suédois » dans la presse française (2000-2020)}

14 En période de cohabitation politique en France, la visite de Jacques Chirac en Suède en 2000 a permis une recrudescence de l'expression «modèle suédois $»^{48}$. Les années 2000-2007 sont marquées dans la presse française par des évocations de politiques publiques suédoises précises que ce soit dans l'éducation, l'égalité des sexes, les relations au travail, la gestion de la prostitution et la confiance dans l'économie et la politique. Les références à l'ancien modèle social-démocrate des années 1960 et 1970 ont quasiment disparu des titres, des éditoriaux ou des articles de fond portant sur le 
modèle suédois. La mutation du modèle suédois a séduit les élites européennes, la bonne réputation de la Suède dans les institutions européennes est largement relayée dans les milieux journalistiques français. Un éditorial de Sébastien Maillard dans $L a$ Croix évoque la mutation du modèle suédois qui a su proposer une "libéralisation de velours »49. Un autre éditorial du 7 octobre 2002 de La Tribune est intitulé «Le modèle suédois fait des envieux partout en Europe $»^{50}$.

Quand on analyse de près la séquence chronologique où l'expression "modèle suédois » apparaît le plus, on se rend compte que 2007-2020 est la période la plus productive avec 1017 apparitions soit $64 \%$ des occurrences. Nul doute que la campagne présidentielle de 2007 a influencé la référence au modèle suédois qui était présente dans la campagne électorale de l'ancienne candidate socialiste Ségolène Royal ${ }^{51}$. L'augmentation des fréquences d'utilisation est aussi liée à la numérisation des journaux qui accentue la visibilité des recherches depuis l'entrée de la société française dans l'ère numérique à la fin des années 1990. Dans la période 2007-2020, de nombreux articles de quotidiens plus centrés sur l'économie s'appuient sur cette expression ( 55 articles de Le Figaro Économie et 55 articles des Échos). Certes, Le Monde reste en tête de classement quand il s'agit d'évoquer ce modèle (84 occurrences) avec notamment un suivi de la politique suédoise et de la présidence suédoise de l'Union européenne qui offre la possibilité à un pays européen pendant six mois de mettre en avant une stratégie pour l'Union européenne ${ }^{52}$. Dans un éditorial du 3 juillet 2009, le titre est « Le modèle suédois peut-il séduire l'Europe? " avec un commentaire soulignant l'évolution de ce modèle ${ }^{53}$. Dans un autre article du 3 juillet 2009, Marion Van Renterghem dresse un portrait paradoxal du Premier ministre de l'époque puisque l'article s'intitule «Le Suédois Fredrik Reinfeldt, un libéral en terre sociale-démocrate $»^{54}$. Ces articles sont révélateurs, car à l'occasion d'une alternance politique, ils donnent l'occasion de décrire la situation suédoise et accentuent l'idée d'un modèle pragmatique ayant évolué ${ }^{5}$.

Les 84 articles du Monde se référant au modèle suédois entre 2007 et 2020 n'ont pas qu'une portée admirative, certains articles font ressortir un modèle paradoxal avec des côtés négatifs. Le correspondant du Monde dans les pays scandinaves et nordiques, Olivier Truc, a consacré des articles plus précis portant sur des phénomènes de société, il utilise le plus souvent le terme de "modèle suédois " pour se référer au modèle social-démocrate fondé sur une industrialisation et une redistribution via l'Étatprovidence ${ }^{56}$. En s'intéressant aux titres mentionnant littéralement l'expression "modèle suédois ", on remarque que l'évolution du nouveau modèle est en proie à un certain nombre de défis qui se posent comme l'immigration, les réformes du marché du travail et de la protection sociale.

Tableau 2 : quelques titres de quotidiens français mentionnant le modèle suédois

\begin{tabular}{|l|l|l|}
\hline Quotidien & Date & Titre \\
\hline Les Échos & 2 décembre 2014 & La Suède à la recherche de son modèle perdu \\
\hline Les Échos & 4 octobre 2016 & $\begin{array}{l}\text { Quand le secteur privé bouscule le «modèle } \\
\text { suédois» }\end{array}$ \\
\hline Le Figaro & 10 février 2016 & Le modèle suédois bousculé par l'afflux de réfugiés \\
\hline
\end{tabular}




\begin{tabular}{|c|c|c|}
\hline L'économie Politique & 1er janvier 2017 & Le modèle suédois est-il toujours social-démocrate? \\
\hline Le Figaro & 31 juillet 2017 & $\begin{array}{l}\text { Macron: le «modèle suédois", une «source } \\
\text { d'inspiration » }\end{array}$ \\
\hline Les Échos & $\begin{array}{ll}17 & \text { novembre } \\
2017 & \end{array}$ & Le modèle suédois toujours gagnant en Europe \\
\hline Alternatives Économiques & $\begin{array}{l}\text { 1er décembre } \\
2017\end{array}$ & Le modèle suédois fait de la résistance \\
\hline Challenges & 4 février 2018 & Le modèle suédois fait rêver, mais s'exporte mal \\
\hline $\begin{array}{l}\text { Sud Ouest / Sud Ouest } \\
\text { Dimanche }\end{array}$ & $\begin{array}{ll}11 & \text { décembre } \\
2019 & \end{array}$ & $\begin{array}{l}\text { Souvent imité, jamais égalé, le modèle suédois est-il } \\
\text { la panacée? }\end{array}$ \\
\hline
\end{tabular}

Source : recherche réalisée sur Nexis Uni (janvier 2020)

17 La plupart de ces titres font mention d'un nouveau modèle en train de se redéfinir parfois sous la pression des événements, d'où l'usage de termes comme «bouscule ». Ces titres identifient le modèle suédois à la social-démocratie avec cette idée qu'il demeure encore inaccessible («s'exporte mal») et exotique à certains égards. La plupart des mentions sont positives à l'instar d'un titre d'article des Échos du 3 juillet 2015 déclarant «Il faut nous inspirer du modèle suédois ${ }^{57}$. Si nous nous intéressons explicitement à l'énonciation du modèle suédois dans le discours journalistique, nous devons prendre en compte la relation entre "modèle suédois ", " modèle scandinave » et « modèle nordique " pour pouvoir apprécier la référence aux transformations de la société suédoise en France ${ }^{58}$.

\section{Le modèle suédois soluble dans un modèle régional}

18 Il semblerait en outre que le modèle suédois soit appréhendé sous un angle plus régional, l'incluant de facto dans un modèle plus large pour mieux effacer ses spécificités. Pour contrôler un biais qui serait lié au repérage de la simple expression "modèle suédois", nous avons également effectué un balayage des titres de presse française évoquant le «modèle nordique » et le "modèle scandinave ». Dans un autre article du Monde datant du 15 avril 2012 et intitulé « Des modèles nordiques soumis à rude épreuve ", Olivier Truc se pose franchement la question : «Le modèle suédois estil encore à la hauteur de sa réputation? $\aleph^{59}$; avec cette fois la mention d'un ensemble de faits marquant l'évolution du modèle (diminution du nombre de fonctionnaires, adaptation à l'économie de marché) ${ }^{60}$. L'approche régionale permet de mettre en perspective le modèle suédois et de comparer ses points forts et ses points faibles. Ainsi, entre 1990 et début 2020, le terme de «modèle nordique » apparaît à 371 reprises dans les quotidiens nationaux et régionaux à l'instar de ce qui est présenté dans le tableau 3. 
Tableau 3 : repérage de l'expression « modèle nordique » dans les quotidiens et magazines français

\begin{tabular}{|l|l|}
\hline Nom du quotidien & Nombre d'articles faisant référence au « modèle nordique » \\
\hline Le Monde & 59 \\
\hline Les Échos & 33 \\
\hline Le Figaro & 24 \\
\hline Agence France Presse & 23 \\
\hline Le Figaro Économie & 18 \\
\hline Le Temps & 18 \\
\hline La Tribune & 11 \\
\hline L'Humanité & 10 \\
\hline
\end{tabular}

Source : recherche réalisée sur Nexis Uni (janvier 2020)

19 La différence avec les mentions du «modèle suédois » vient du fait que les quotidiens mentionnent en moyenne quatre fois moins le «modèle nordique». Cet écart s'accentue avec les quotidiens régionaux qui utilisent très peu cette référence (respectivement 9 et 6 occurrences pour Ouest-France et Sud Ouest / Sud Ouest Dimanche). En revanche, on constate également une augmentation de la fréquence à partir de 2006 puisqu'entre 2006 et 2020, il y a 294 mentions de ce modèle soit $79 \%$ des occurrences pour cette période. Pour la presse française, il est plus aisé de commenter un modèle culturel que d'avoir une approche comparée en resituant la Suède dans son ensemble régional.

Dans la multiplication des références aux modèles nordiques, que ce soit d'un point de vue régional ou d'un point de vue national, les articles insistent sur la banalisation de ce modèle. Il existe ainsi un déplacement mythologique du modèle suédois vers le modèle nordique qui a été mis à l'encan par les journalistes. Dans l'analyse globale des articles mentionnant le "modèle nordique», les personnalités politiques les plus associées à l'expression sont Emmanuel Macron (23 occurrences), Ségolène Royal (20 références) et François Hollande (15 mentions). Autrement dit, les journalistes ont tendance à reprendre les propos de ces trois personnalités sur le "modèle nordique " alors que pour le "modèle suédois ", Emmanuel Macron était davantage cité. Pour ce dernier, la visite d'État au Danemark en août 2018 a été l'occasion d'une série de commentaires sur la perception du "modèle nordique» dans la foulée de ses commentaires sur le modèle suédois délivrés lors de l'été 2017. Un article du 28 août 2018 des Échos est d'ailleurs intitulé « Europe : Macron à la recherche du modèle nordique $»^{61}$. Dans une interview du 7 juillet 2017, François Villeroy de Galhau, gouverneur de la Banque de France depuis 2015, adressait quelques conseils au nouveau président élu au sujet de la réforme du marché du travail : « Si on le compare au modèle nordique, notre droit du travail protège trop les emplois, mais pas assez les personnes. Et nous faisons trop confiance à des normes complexes et pas assez au dialogue social $\aleph^{62}$. En d'autres termes, le modèle nordique est identifié à un lexique social 
libéral, où les réformes économiques sont possibles avec une souplesse de la protection sociale et des normes qui sont renégociées entre les partenaires sociaux.

21 L'analyse des titres est également révélatrice de la manière dont le modèle nordique est affiché. Le tableau 4 présente cette fois quelques titres des quotidiens comportant l'expression « modèle nordique ».

Tableau 4 : quelques titres de quotidiens et magazines français mentionnant le modèle nordique

\begin{tabular}{|l|l|l|}
\hline Quotidien & Date & Titre \\
\hline $\begin{array}{l}\text { Ouest- } \\
\text { France }\end{array}$ & 19 mars 2007 & Dominique Méda : Un modèle efficace, le nordique \\
\hline Les Échos & 11 octobre 2010 & Les lumières du modèle nordique \\
\hline Le Monde & 3 février 2012 & La refonte du modèle nordique se prépare en Suède \\
\hline Le Monde & $27 \quad$ novembre & Le modèle nordique fait toujours autant rêver l'Europe \\
\hline Challenges & 8 décembre 2016 & L'école finlandaise, l'autre modèle nordique à suivre \\
\hline Le Monde & 8 novembre 2018 & La face cachée du modèle nordique \\
\hline Wansquare & 22 mai 2019 & $\begin{array}{l}\text { Divergence Suède - Norvège : le modèle nordique peut-il } \\
\text { survivre? }\end{array}$ \\
\hline Nord Éclair & 25 août 2019 & On vante souvent les modèles nordiques... \\
\hline
\end{tabular}

Source : recherche réalisée sur Nexis Uni (janvier 2020)

22 Les titres font apparaître tantôt la diversité des modèles nordiques tantôt leurs ressemblances. Mis à part quelques titres plus négatifs portant sur une politique publique spécifique rejetée, les mentions du modèle insistent sur la dimension du renouveau (« refonte »). En revanche, lorsque l'on repère les articles citant le « modèle scandinave ", nous recensons 712 occurrences entre 1990 et 2020 avec une fréquence plus élevée à partir de l'année 2005. Nous retrouvons le même classement avec Le Monde, Les Échos, Le Figaro puis des quotidiens comme La Croix (21 occurrences) et des quotidiens régionaux tels que Sud Ouest / Sud Ouest Dimanche (19 mentions), Ouest-France (16 mentions), La Voix du Nord (15 références) et La Montagne (14 références). Ab hinc, l'exemple de la Suède est souvent cité dans les articles affichant le «modèle scandinave ». Dans un article publié dans L'Obs le 8 octobre 2017, l'économiste Philippe Aghion est interviewé et évoque les réformes fiscales de Macron qui s'inspirent du modèle suédois ${ }^{63}$. Le « modèle suédois » sert ainsi d'étalon justifiant des réformes de profondeur garantissant une protection sociale adaptée. Même s'il existe des références au "modèle danois » et au "modèle finlandais », le «modèle suédois » apparaît à de nombreuses reprises lié à l'invocation du « modèle scandinave ${ }^{64}$. Comme l'écrivait Roger de Weck, « on a vanté le modèle japonais, voire le modèle soviétique puis le modèle rhénan avant de s'enthousiasmer pour le modèle suédois, l'américain ou 
le chinois! En un demi-siècle, tous ces modèles ont été portés aux nues puis délaissés ${ }^{65}$.

\section{Conclusion}

Les usages du " modèle suédois » dans les quotidiens et magazines français révèlent des perceptions qui ont évolué entre 1990 et 2020. Le début des années 1990 est marqué par des références ambivalentes à l'ancien modèle social-démocrate en crise au moment où la Suède s'apprête à entrer dans l'Union européenne. Le modèle suédois incarné par un parti social-démocrate hégémonique disparaît peu à peu des références pour laisser place à un "nouveau modèle suédois " porté sur l'évolution d'un compromis entre l'État-Providence et les forces du marché. Les années 2000 sont un tournant dans la presse française avec la multiplication d'éditoriaux et d'articles de fond traitant des politiques publiques précises ${ }^{66}$. Les références négatives y sont présentes, mais grosso modo l'image positive de la Suède traverse les grands quotidiens pour mieux faire ressortir la crise sociale et économique de la France au lendemain du référendum de 2005. La campagne présidentielle de 2007 a permis de banaliser les références à ce modèle dans la presse française. Désormais, le modèle suédois est synonyme d'adaptation à une mondialisation heureuse avec un fort système de protection sociale. L'invocation directe du « modèle suédois » est également mobilisée dans les références aux modèles scandinaves et nordiques dans les quotidiens et magazines français. Même si le modèle danois est également largement commenté, c'est le modèle suédois qui demeure au cœur de ces références françaises et qui sert de boussole à la définition du modèle régional. Par extension métaphorique, le modèle nordique semble s'extraire davantage de la référence au modèle suédois dans la presse française. La présente recherche appelle des travaux complémentaires sur la présentation du modèle suédois dans les émissions télévisées et radiophoniques pour avoir une vue d'ensemble sur sa diffusion récente en France. De plus, une comparaison diachronique avec les usages antérieurs de ce modèle dans la presse française serait nécessaire. Il serait également intéressant de comparer les emprunts aux modèles danois, norvégien et finlandais pour pouvoir apprécier la fascination exercée par le modèle scandinave et nordique. In fine, la description d'un modèle ne peut pas se limiter à la mention explicite du terme "modèle ", il est donc important de pouvoir aussi analyser dans la presse française les références à la société suédoise pouvant refléter une certaine tendance à l'essentialisation d'un éthos culturel.

\section{NOTES}

1. Jenny Andersson, «Qu'est-ce que ce modèle suédois? Débats constitutifs pour comprendre l'histoire de la social-démocratie suédoise, son modèle et son évolution jusqu'aux élections de septembre 2010 », Histoire@Politique. Politique, culture, société, 13, janvier-avril 2011, p.161-175. Kasimierz Musial, Roots of the Scandinavian Model: Images of Progress in the Era of Modernisation, 
Baden-Baden, Nomos, 2002. La gauche française a commencé à référencer ce modèle au début des années 1990 à l'instar du PS présentant une motion en 1993 nommant ce modèle (Michel Lecluse, "Motion préparatoire au congrès d'octobre - Réduction du temps de travail, libre-échange : les interrogations des socialistes ", Les Échos, 16 juillet 1993, 677 mots). Le journaliste d'origine hongroise, Ferenc Fejtö, avait consacré un chapitre sur «le modèle suédois » dans son analyse complète du réformisme social-démocrate et après avoir eu des entretiens avec Olof Palme. Ferenc Fejtö, La social-démocratie quand même : un demi-siècle d'expériences réformistes, Paris, Laffont, 1980 , p. 75.

2. https://www.elysee.fr/emmanuel-macron/2017/11/17/conference-de-presse-bilaterale-demmanuel-macron-et-de-stefan-lofven-premier-ministre-suedois (consulté le 15 janvier 2020).

3. Voir l'exemple d'article présentant le modèle à suivre en termes de réformes et de contraintes. «Retraites: une réforme systémique sur le modèle suédois est nécessaire ", Économie Matin, 12 décembre 2019, 2078 mots. Voir également Christophe Boutin, «Réforme des retraites : et maintenant? ?, La Nouvelle République du Centre Ouest, 7 janvier 2020, 622 mots.

4. Emile Chabal, A Divided Republic, Nation, State and Citizenship in Contemporary France, Cambridge, Cambridge University Press, 2015, p. 1-6.

5. Pierre Rosanvallon, Le modèle politique français, Paris, Seuil, 2004.

6. Yohann Aucante, «Den 'svenska modellen' i fransk samhällsvetenskap : en översikt », dans Mickaëlle Cedergren, Sylvain Briens (dir.), Médiations interculturelles entre la France et la Suède, Trajectoires et circulations de 1945 à nos jours, Stockholm, Stockholm University Press, 2015, p. 27.

7. Yohann Aucante, op. cit., p. 28.

8. Yohann Aucante, «La chasse au modèle : l'État social suédois en science politique », Raisons politiques, 2002/2, $n^{\circ} 6$, p. 119.

9. Il est intéressant de noter que les médiateurs du «modèle suédois » dans ces années sont le plus souvent des journalistes et des militants. Il existe des références dans la presse quotidienne à ce modèle suédois. Andreas Hellenes a montré également le rôle de la diplomatie culturelle suédoise en France dans les efforts de promotion de ce modèle. Andreas Hellenes, «Culture et information. Le Centre culturel suédois et sa création », dans Mickaëlle Cedergren, Sylvain Briens (dir.), Médiations interculturelles entre la France et la Suède, Trajectoires et circulations de 1945 à nos jours, Stockholm, Stockholm University Press, 2015, p. 100.

10. Edgar Morin, « Les maîtres mots », $n^{\circ} 13$, Revue du M.A.U.S.S., $3^{\mathrm{e}}$ trimestre, 1991, p. 33.

11. Jenny Andersson, «Qu'est-ce que ce modèle suédois? Débats constitutifs pour comprendre l'histoire de la social-démocratie suédoise, son modèle et son évolution jusqu'aux élections de septembre 2010 », Histoire@Politique. Politique, culture, société, 13, janvier-avril 2011, p. 168.

12. Christopher S. Browning, Antonio Ferraz de Oliviera, «Introduction : Nation Branding and Competitive Identity in World Politics », Geopolitics, 2017, vol. 22, n 3, p. 483.

13. Roland Barthes, Mythologies, Paris, Seuil, 1957, p. 240.

14. Cornelius Castoriadis, L'institution imaginaire de la société, Paris, Seuil, 1975, p. 359-360.

15. Cornelius Castoriadis, L'institution imaginaire de la société, Paris, Seuil, 1975, p. 434.

16. Gilles Vergnon, Le "modèle suédois": Les gauches françaises et l'impossible social-démocratie, Rennes, Presses universitaires de Rennes, 2015.

17. Gösta Bagge, Politiska tal, 1936, Anförande i radio den 4 september 1936. Cette référence nous a été communiquée par Nils Edling qui effectue un travail d'histoire conceptuelle autour des métaphores de l'État-providence. Voir Ragnar Björk, Bert Edström, Thomas Lundén, Rudolf Kjellén: geopolitiken och konservatismen, Stockholm, Hjalmarson \& Högberg, 2014. Selon Nils Hedling, il existait déjà une panoplie de références à la métaphore du folkhem bien avant Rudolf Kjellén. Henrik Björck, Folkhemsbyggare, Stockholm, Atlantis, 2008. Voir également la thèse d'Andreas Mørkved Hellenes, Fabricating Sweden. Studies of Swedish Public Diplomacy in France from the 1930s to the 1990s, Université d'Oslo, Sciences Po Paris, 2019. 
18. Michel Espagne, «La notion de transfert culturel», Revue Sciences / Lettres, 1, 2013. URL : http://journals.openedition.org/rsl/219

19. Martin Kylhammar, Bland resenärer och kosmopoliter, Stockholm, Carlsson Bokförlag, 2017, p. 24. Martin Kylhammar, « Comment le modèle suédois est né en France. Les récits de voyage en Suède de Serge de Chessin ", dans Mickaëlle Cedergren, Sylvain Briens (dir.), Médiations interculturelles entre la France et la Suède, Trajectoires et circulations de 1945 à nos jours, Stockholm, Stockholm University Press, 2015, p. 16-23.

20. Ibid., p. 27. Serge de Chessin, Les Clefs de la Suède, Paris, Hachette, 1935. Jean-François Battail, Régis Boyer, Vincent Fournier, Les sociétés scandinaves de la Réforme à nos jours, Paris, Presses universitaires de France, 1992. Il faudrait aussi rappeler le message optimiste rapporté par Émile Schreiber dans son ouvrage Heureux Scandinaves publié en 1937. Émile Servan-Schreiber, Heureux Scandinaves, enquête sur les réalisations socialistes au Danemark, en Suède, Norvège et Finlande, Paris, Denoël et Steele, 1937. Voir Yohann Aucante, «Den 'svenska modellen' i fransk samhällsvetenskap : en översikt », dans Mickaëlle Cedergren, Sylvain Briens (dir.), Médiations interculturelles entre la France et la Suède, Trajectoires et circulations de 1945 à nos jours, Stockholm, Stockholm University Press, 2015, p. 26.

21. Christian de Caters, Visages de la Suède, Paris, Plon, 1930.

22. Émile Servan-Schreiber, Heureux Scandinaves, enquête sur les réalisations socialistes au Danemark, en Suède, Norvège et Finlande, Paris, Denoël et Steele, 1937. Paul Planus, Patrons et ouvriers en Suède, Paris, Plon, 1938.

23. Martin Kylhammar, Bland resenärer och kosmopoliter, Stockholm, Carlsson Bokförlag, 2017, p. 25 .

24. Jean Parent, Le modèle suédois, Paris, Calmann-Lévy, 1970. Voir aussi Gilles Coulombe, «Relations du travail dans le secteur public. Le modèle suédois : mythe ou réalité », Relations industrielles, vol. 31, n. 3, 1976, p. 448-465.

25. Guy de Faramond, Une politique du bien-être, Paris, Seghers, 1972 ; Guy de Faramond, La Suède et la qualité de la vie, Paris, Le Centurion, 1975. Gabriel Ardant, La révolution suédoise, Paris, Robert Laffont, 1976. Guy de Faramond, Claude Glayman, Suède. La Réforme permanente, Paris, Stock, 1977. Voir Yohann Aucante, op. cit., p. 27. La thèse d'Andreas Hellenes est centrée sur cette construction discursive. Andreas Mørkved Hellenes, Fabricating Sweden. Studies of Swedish Public Diplomacy in France from the 1930s to the 1990s, Université d'Oslo, Sciences Po Paris, 2019.

26. Yves Bourdet, "La Suède et les limites de l'État-providence ", Revue de l'OFCE, 1988, 25, p. 75-100. Il y a également des publications en français de chercheurs suédois. Lasse Krantz, "L'anthropologie au service du développement international : Un modèle suédois ", Anthropologie et Sociétés, vol. 8, n³, 1984, p. 79-93. Pour sa part, Élisabeth Elgan s'interroge l'idée d'un modèle suédois fondé sur le dialogue. Elisabeth Elgan, «Le printemps 1968 en Suède ", Matériaux pour l'histoire de notre temps, 1988, p. 56-59.

27. Jacques Arnault, Le modèle suédois revisité, Paris, L'Harmattan, 1992. Le témoignage de Jacques Arnault est intéressant car il s'appuyait sur une précédente étude de 1970. Jacques Arnault, Le socialisme suédois, Paris, éditions sociales, 1970. Jacques Arnault, dirigeant communiste, syndicaliste et journaliste à L'Humanité, témoigne de la pénétration de cette fascination dans les milieux communistes. D'autres travaux commencent à s'intéresser à des politiques spécifiques en Suède. Nasr-Eddine Malamane, «Intégration, l'autre 'modèle suédois' ", Hommes \& Migrations, 1992, p. 21-27.

28. On citera ici les travaux de Nathalie Morel. Nathalie Morel, « Le modèle universaliste suédois au prisme du libre choix », Lien social et Politiques, 2011, 66, p. 139-154. Nathalie Morel, «L'Étatprovidence suédois comme modèle social productif », Revue des politiques sociales et familiales, 2013, 112 , p. 39-49. Bruno Palier, «De la crise aux réformes de l'État-providence. Le cas français en perspective comparée ", Revue française de sociologie, 2002, 43-2, p. 243-275. 
29. Magnus Falkehed, Le modèle suédois: santé, services publics, environnement: ce qui attend les Français, Paris, Payot, 2003. En revanche, dans l'ouvrage d'Alain Lefebvre et de Dominique Méda publié en 2006, le modèle nordique est largement plébiscité dans l'équilibre entre mondialisation économique et protection sociale. Voir Alain Lefebvre, Dominique Méda, Faut-il brûler le modèle social français ?, Paris, Seuil, 2006.

30. Alain Lefebvre, Macron le suédois, Paris, PUF, 2018.

31. Pierre Muller, Les politiques publiques, Paris, PUF, 1990, p. 64.

32. Antoine Blanc, Hélène Peton et Frédéric Garcias, «L'analyse lexicométrique des macro discours par les vocabulaires - enjeux théoriques et méthodologiques ", Finance Contrôle Stratégie [En ligne], NS-6|2019, mis en ligne le 23 mai 2019, consulté le 03 avril 2020. URL: https://journals.openedition.org/fcs/3459 ; DOI : https://doi.org/10.4000/fcs.3459

33. Section étranger, «Le modèle suédois malmené », Le Monde, 17 février 1990, 318 mots.

34. Éric Le Boucher, «La fin du rêve suédois ", Le Monde, 14 septembre 1991, 1042 mots.

35. Éric Le Boucher, " Privatisations suédoises », Le Monde, 13 novembre 1991, 600 mots.

36. Françoise Nieto, «L'un des pères du modèle social-démocrate, l'ancien ministre Gunnar Strang, est mort », Le Monde, 10 mars 1992, 292 mots.

37. Rolf Gustavsson, «Le 'chauvinisme du bien-être' à l'épreuve en Suède », Le Monde, date, 1023 mots.

38. Jean-Pierre Durand, Christian Berggren (dir.), La fin du modèle suédois, Paris, Syros.

39. Claude Juvenal, « Renault-Volvo : l'alliance de deux cultures ouvrières d'avant-garde », Agence France Presse, 6 septembre 1993, 1339 mots. Sur le périmètre de cette alliance stratégique, voir Desalegn Abraha, Syeda-Masooda Mukhtar, «Strategic Alliance Breakups: the Volvo-Renault lesson ", in N. J. Delener, Leonora Fuxman, F. Victor Lu, Susana Rodrigues (eds.), Exploring the possibilities for sustainable future growth in business and technology management, Global Business Technology Association, 2015, p. 1-20.

40. Maurice Snyders, «La Suède en quête d'une crédibilité européenne - La Suède est condamnée à reconstruire son modèle », Les Échos, 27 mars 1995, 1433 mots.

41. Auteur, « La fin du modèle suédois », Le Monde, 7 avril 1995, 171 mots.

42. Alain Debove, «La social-démocratie a retrouvé le pouvoir en Europe du Nord », Le Monde, 7 avril 1995, 2107 mots.

43. Pierre-Alain Furbury, "Protection sociale - Le "modèle suédois" à l'épreuve ", Les Échos, 9 novembre 1995, 537 mots. Cette perception du mythe suédois avait été étudiée dans la recherche. Lena Sanders, «L'Europe s'arrête-t-elle à la Baltique?», Espace géographique, 1990, 19-20, p. 97-104.

44. Jean-Luc Chandelier, "M. Persson appelle à créer un 'nouveau modèle' suédois ", Agence France Presse, 16 mars 1996, 583 mots.

45. Section Monde, « Le pays où les femmes sont reines », Le Point, 6 avril 1996, 2611 mots.

46. Catherine Leroy, « Les négociations se décentralisent en Suède », Journal, 19 mars 1997, 1600 mots.

47. Cet éditorial de Laurent Dominati dans le Figaro intitulé ironiquement «Le "modèle suédois ", se fait un plaisir de révéler cette face sombre, le modèle social-démocrate suédois est ainsi comparé au modèle yougoslave. Le Figaro, 15 septembre 1997, 574 mots.

48. Jean-François Bège, Sud Ouest, 12 avril 2000, 361 mots (éditorial).

49. Sébastien Maillard, «La Suède se pose à nouveau en modèle économique et social », La Croix, 13 septembre 2002, 596 mots, p. 8 .

50. Jacques Roucourel, «Le modèle suédois fait des envieux partout en Europe ", La Tribune, 7 octobre 2002, 589 mots, p. 8 .

51. Benjamin Huteau, Jean-Yves Larraufie, Le modèle suédois, un malentendu? Paris, Presses des Mines, 2009, p. 7. 
52. Anna Stellinger, «La présidence suédoise de l'Union européenne en pleine mutation », Nordiques, $\mathrm{n}^{\circ} 23$, automne 2010, p. 52.

53. Éditorial, «Climat: le modèle suédois peut-il séduire l'Europe?", Le Monde, 3 juillet 2009, p. 1 (201 mots).

54. Marion Van Renterghem, «Le Suédois Fredrik Reinfeldt, un libéral en terre socialedémocrate ", Le Monde, 3 juillet 2009, p. 10 (992 mots).

55. Ibid.

56. Les correspondants des grands quotidiens nationaux ont en réalité une importance capitale dans l'utilisation de cette notion de modèle, même si la présentation y est nuancée. Ils jouent le rôle de passeurs pour relayer une image de la Suède et des pays scandinaves et nordiques en France.

57. Pierre de Gasquet, "Il faut nous inspirer du modèle suédois ", Le Monde, 3 juillet 2015, 717 mots.

58. Cette démarche vise en réalité à expliciter et à affiner la perception de ce modèle dans la mesure où il existe une grande labilité dans les références au modèle nordique. Voir Robert Cox, "The Path-dependency of an Idea: Why Scandinavian Welfare States Remain Distinct, Social policy and administration ", Social policy and Administration, 2004, vol. 38, 2, p. 204-219.

59. Olivier Truc, "Des modèles nordiques soumis à rude épreuve ", Le Monde, 15 avril 2012, p. 5 (844 mots).

60. Ibid.

61. Jacques Hubert-Rodier, "Europe : Macron à la recherche du modèle nordique ", Les Échos, 28 août 2018, 656 mots. La visite d'État du Président Macron avait largement été demandée par l'ancien ambassadeur de France au Danemark, François Zimeray, qui voyait dans le Danemark un modèle recherché par la France. Voir l'article Antoine Jacob, « Le Danemark, un pays modèle en plein questionnement », La Croix, 17 février 2016.

62. Propos recueillis par Marie Charrel et Véronique Chocron, «Je ne crois pas à la France bloquée ", Le Monde, 7 juillet 2017, p. 4, 1404 mots.

63. Sophie Fay, «La théorie du ruissellement est une fable, pas le modèle scandinave! ", L'Obs, 8 octobre 2017, 1283 mots.

64. Dans la base de données Nexis Uni, le « modèle danois » apparaît à 774 plusieurs reprises avec un pic entre les attentats de Copenhague en 2015 et la visite du Président Macron à l'été 2018. Le «modèle finlandais» est mentionné quant à lui à 261 reprises dans les quotidiens français à partir de 1997 ; ce dernier est moins appréhendé globalement, il est évoqué selon des politiques publiques précises (éducation, nucléaire...). La notion de « modèle finnois » n'apparaît qu'une fois dans un article du Sud Ouest datant du 27 novembre 2007.

65. Roger de Weck, Les Échos, 10 janvier 2007, n 19832, p. 1.

66. Benjamin Huteau, Jean-Yves Larraufie, Le modèle suédois, un malentendu? Paris, Presses des Mines, 2009, p. 13.

\section{RÉSUMÉS}

L'objectif de l'article est de montrer comment les références au modèle suédois ont été mobilisées ces dernières années dans la presse française. Ainsi, au-delà du discours politique, les analyses journalistiques des mentions du modèle suédois depuis les années 1990 dans les 
quotidiens et magazines français permettent de comprendre la manière dont le grand public s'est intéressé à la situation des pays scandinaves et nordiques. Les grands quotidiens multiplient ces mentions à partir du début des années 2000 sur fond de découverte du "nouveau modèle suédois ». Si des critiques de ce modèle se glissent dans l'appréciation de certaines politiques publiques spécifiques, le modèle est valorisé globalement sous son aspect fonctionnaliste et pragmatique.

The aim of the article is to show how references to the Swedish model in the French press have been used in the recent. Beyond political discourse, the analysis of these references since the 1990s in French newspapers and magazines help to understand the way a broader public has begun to get interested in the situation of Scandinavian and Nordic countries. The daily newspapers have multiplied these mentions from the beginning of 2000 when they adhered to the emergence of a "new Swedish model ». If critics of this model appear in the appreciation of some specific public policies, the model is on the whole perceived under its functionalist and pragmatic aspect.

\section{INDEX}

Mots-clés : modèle suédois, mythologie, presse française, imaginaire, réformes économiques

Keywords : Swedish model, liberal narative, French press, mythology, imaginary

\section{AUTEUR}

\section{CHRISTOPHE PREMAT}

maître de conférences, Université de Stockholm 